\title{
Longitudinal vehicle dynamics model for construction machines with experimental validation
}

\author{
A. Alexander* and A. Vacca \\ Mechanical Engineering, Purdue University \\ 1500 Kepner Dr., Lafayette, IN, 47905, USA \\ *Email: addisonalexander@purdue.edu \\ Phone: +17654471609; Fax: +17654481860
}

\begin{abstract}
Construction machines represent a particular set of difficulties when modelling their system dynamics. Due to their generally low velocities and unorthodox operating conditions, the standard modelling equations used to simulate the behaviour of highway vehicles can have a poor behaviour for these systems. This paper sets forth a vehicle model which is suitable for construction machines, which travel at low velocities and encounter significant external forces in daily operation. It then shows the work done in validating the machine model with experimental data. First, the overall vehicle dynamics are developed, including a model for the machine behaviour when pushing against a resistive force. Then, a wheel force generation model suitable for low-velocity systems is discussed. Finally, pertinent experimental results are presented. Two different model validation tests were run. Both tests generated results which were matched well by the simulation model. In fact, the model matches experimental data reasonably well for both roading and pushing conditions. This indicates that the modelling methods described in this work are appropriate for the modelling of low-velocity systems such as wheel loaders and other construction machinery.
\end{abstract}

Keywords: Vehicle dynamics, dynamic system modelling, traction control, construction machinery

\section{INTRODUCTION}

Vehicle dynamics models for on-road machines have been around for quite some time and are relatively well understood. On the other hand, vehicle systems in other areas have not enjoyed the same level of interest; therefore, a standardised dynamics modelling method has not been established. The particular behaviour of construction machines requires some further considerations which differentiate them from standard passenger vehicles. Some previous work has been done in modelling these systems, including component modelling work by Wong [1] and Andreev [2], with even more investigations recently [3,4]. Choosing a vehicle dynamics modelling strategy is often heavily dependent on the application at hand. Many different dynamic models exist for describing aspects of the vehicle suspension system [5] or various resistances [6]. Instead, this work focuses on modelling the driveline and wheels of construction machines. Different approaches to the problem of the wheel and tire dynamics modelling have been done in the past, each incorporating different idea or physical models [7-10]. Most common modern methods stemmed from variations on the seminal work done by Pacejka [11]. 
This is perhaps the most widely used tire force model in vehicle dynamics literature and simulation software. Its relatively simple formulation makes it a good choice for implementing into system models but also causes numerical issues when addressing systems which fall outside the normal conditions for vehicle simulations. As construction machines are primarily driven at low velocities where the standard wheel dynamics equations become unstable, these equations must be modified to allow simulations to run effectively. Various works have presented different methods of accounting for this behaviour. Pacejka himself presents one such method [12], while another was set forth by Bernard [13]. The work done in this research builds on the foundation laid by Bernard.

This research also includes an experimental investigation into the characterisation of tires used on construction equipment. These tires are much larger than passenger vehicle tires, and they are designed with other characteristics in mind, so their force generation characteristics can be quite different than those of typical on-road tires. In order to determine the slip-friction profile of the construction machine tires, a series of tests were run by using a state observer to estimate the force at each wheel. This method is partially based on the work done by Rajamani [14]. The purpose of this paper is to formulate a vehicle dynamics model suitable to study the typical low-velocity operation of construction machines. Starting from the torque provided by the engine, the model determines both the vehicle and the wheel velocities, accounting for the behaviour of the mechanical powertrain transmission system and the tire-ground interaction. This model improves on previous work in the field by incorporating considerations which allow for simulations of standard operating modes, such as pushing and digging at low speed. The model is then validated by comparing it against experimental data.

\section{METHODS AND MATERIALS}

To verify the usefulness of the simplified model, testing was conducted to show the simulated results as compared to real-world data. First, a typical vehicle model for straight-line motion is described, including considerations for low-velocity motion and the effect of resistive forces opposing the machine motion. This model includes wheel and body kinematics, as well as a differential slip-friction model. Some methods used to overcome some of these model limitations for the case of construction machinery. Then contains descriptions of test setups and corresponding data which were used to assess the suitability of the vehicle model. The reference machine used for this research is a fourteen-metric ton wheel loader with a bucket which can hold up to $2.3 \mathrm{~m}^{3}$ of material (Figure 1). This machine has been used within the authors' research group in other research activities related to controlling the motion to reduce cab vibrations [15]. In order to validate the models created for simulating the system, the slip-friction characteristics of the wheel loader tires needed to be determined. To that end, a method was implemented using a state observer to estimate the force at each tire. This estimator uses measurements of the machine's linear velocity, the four-wheel velocities, and the acceleration of the vehicle in order to make this force estimation. Then, tests were conducted to validate the simplified resistive force model used in simulations. These tests required an estimate of the total pushing force of the machine. To achieve this estimate, the pressure in the main boom cylinders of the wheel loader, as well as the angle of the boom, were measured and then converted into force using geometric constraints. The sensors needed to take these measurements are represented in Figure 1. Furthermore, data from the wheel loader's CAN bus, including engine torque and speed, were recorded in order to better define each test. 


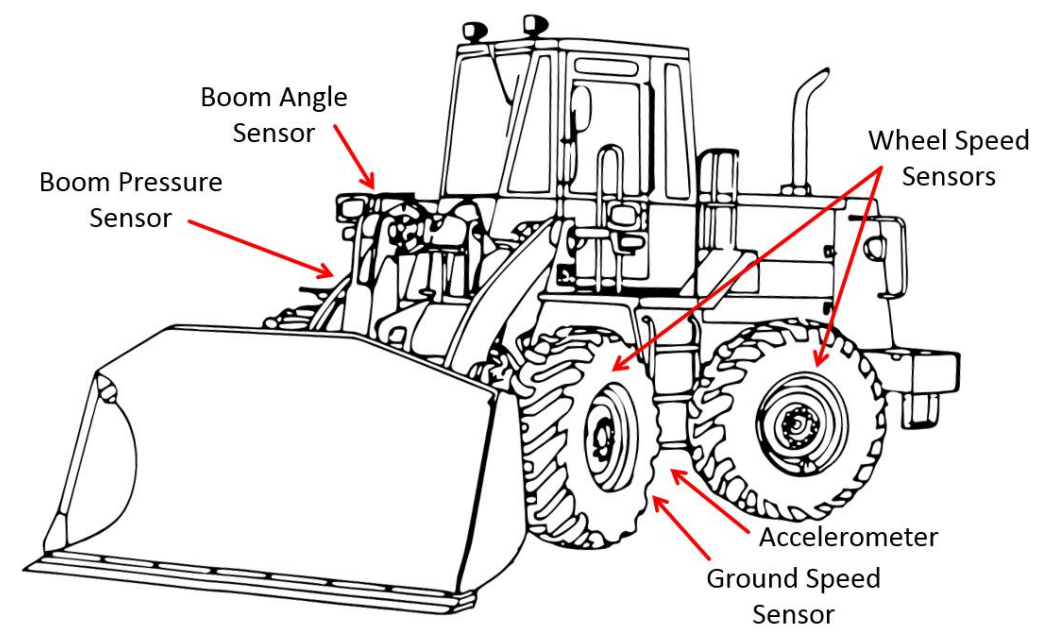

Figure 1. Wheel loader with instrumentation.

\section{Vehicle Dynamics Modelling}

The general model of the wheel loader system for studying linear motion and the effects of longitudinal forces on the system are discussed. A similar method for on-road vehicles was developed and validated by Ahmad [16]. A schematic of the overall system model is shown in Figure 2.

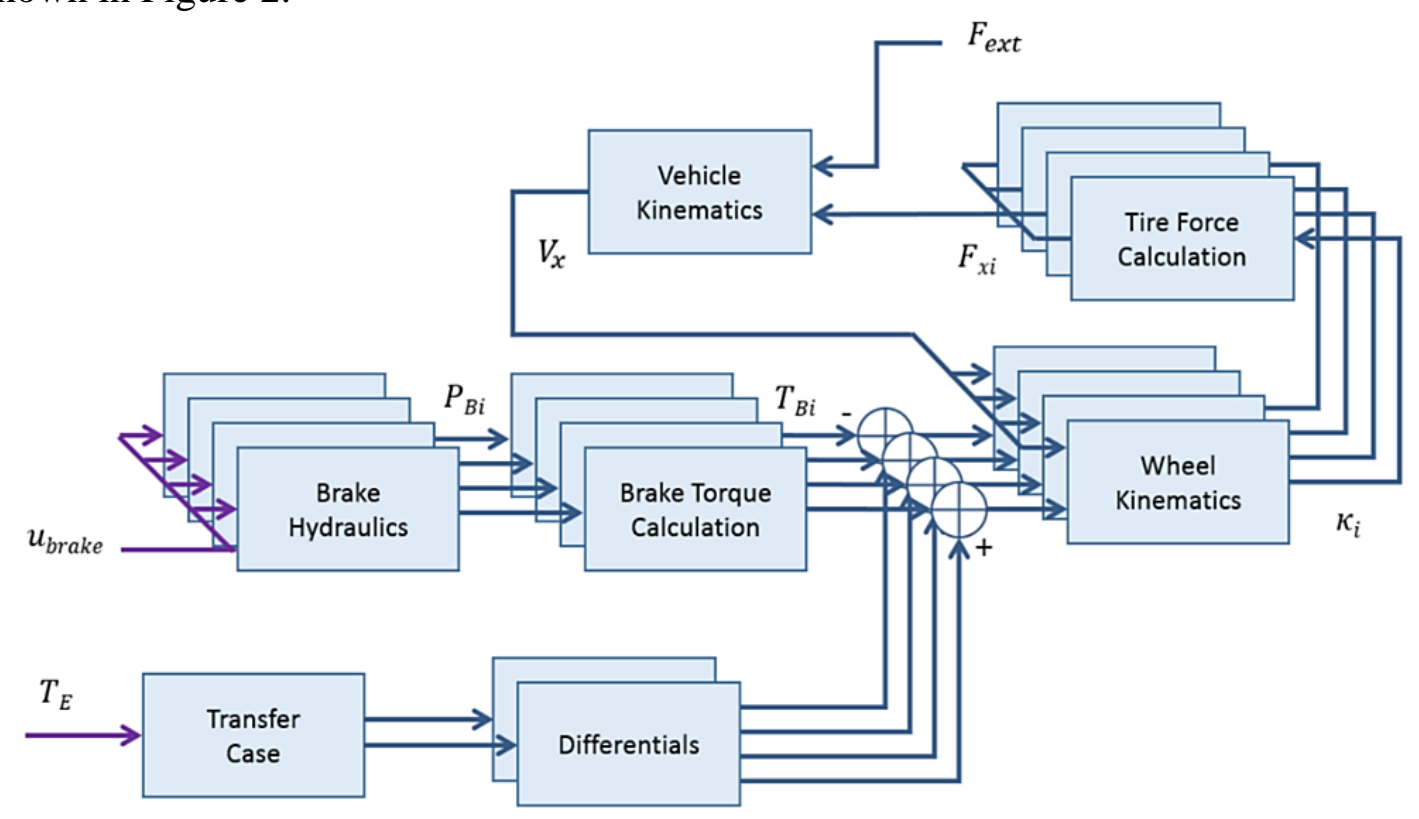

Figure 2.Vehicle dynamics model schematic.

\section{Quarter-Car Vehicle Model}

The first step is to develop a proper vehicle dynamics model known as a quarter-car model, wherein the system is represented as a single mass sitting atop a single wheel (see Figure 3). This model only considered the longitudinal motion of the vehicle (i.e. toward the front or rear of the machine), so that what is left is a simple formulation of machine dynamics. For discussions on lateral forces and moments not included in this model, see [17-19]. When the system is laid out in such a configuration, the dynamics are described 
simply by doing a force and torque balance on the chassis and wheel, respectively [20]. This leaves the following equations for describing the system behaviour:

$$
\begin{gathered}
m \dot{v}_{x}=F_{x}-F_{\text {roll }} \operatorname{sgn}\left(v_{x}\right)-F_{\text {resist }} \operatorname{sgn}\left(v_{x}\right) \\
I_{w} \dot{\omega}=T_{E}-T_{B} \operatorname{sgn}(\omega)-r_{d} F_{x}
\end{gathered}
$$

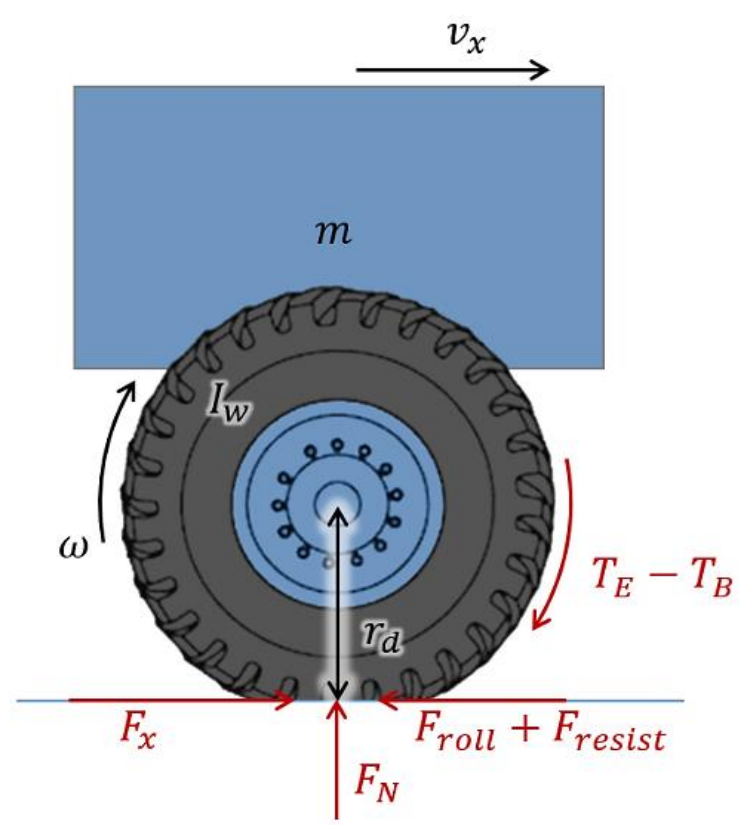

Figure 3. Quarter-car vehicle model.

In Equation (1), $m$ is the mass of the chassis, $v_{x}$ is the longitudinal velocity of the vehicle, $F_{x}$ is the longitudinal tractive force generated by the wheel, $F_{\text {roll }}$ represents the rolling resistance of the machine, $F_{\text {resist }}$ is the other resistive forces. Resistive forces include air resistance, resistance from driving on an incline, and other forces which can act against the motion of the vehicle (of course, in the proper conditions, these can become assisting forces as well). For the purposes of this research, rolling resistance was considered constant, while other resistances (such as air resistance) were neglected due to the fact that the system is always at relatively low velocities. These simplifications are well in line with standard vehicle dynamics modelling practices [1, 20, 21]. More information on complex modelling of rolling resistance can be found in [12] and [22]. Other works have included further resistances, including those stemming from the deformation of soil at the tire-ground interface [23, 24]. Equation (2) includes $I_{w}$, the moment of inertia of the wheel; $T_{E}$, the engine torque being applied on the wheel; $T_{B}$, the braking torque; and $r_{d}$, the dynamic radius of the wheel. The dynamic radius represents the distance between the centre of the wheel and ground when the wheel is in motion, and its value is between that of the tire's unloaded radius and its static loaded radius. In this work, $r_{d}$ is calculated by using the method from Jazar [22]. When incorporated into the full vehicle model, Equation (1) remains more or less the same, as the chassis is modelled as a single mass; however, Equation (2) is copied for use with each of the four wheels. Therefore, these two equations become five equations: one for the linear chassis dynamics and four for the rotational wheel dynamics. 


\section{Two-Axle Vehicle Model}

The longitudinal tractive force from each tire is dependent on the normal force at that wheel. Thus, it is important to accurately model the normal forces. In order to describe how the normal force on each tire changes over time, it is possible to simply treat each axle as its own contact point with the road surface with the effect of both tires on a given axle rolled into a single point force. Figure 4 shows a schematic of the system and highlights the important forces which act on the machine in a normal straight-line operation. By doing a force and moment balance similar to that in [22], the normal force at each wheel can be found.

$$
\begin{aligned}
& F_{z 1}=\frac{1}{2} m g \frac{l_{2}}{l}-\frac{1}{2} m a \frac{h_{C G}}{l}-F_{p u s h} \frac{h_{p}}{l} \\
& F_{z 2}=\frac{1}{2} m g \frac{l_{1}}{l}+\frac{1}{2} m a \frac{h_{C G}}{l}+F_{p u s h} \frac{h_{p}}{l},
\end{aligned}
$$

where $F_{z 1}$ and $F_{z 2}$ are the normal forces at each wheel on the front and rear axles, respectively, $m$ is the mass of the machine, $l_{1}$ and $l_{2}$ are the horizontal distances from the centre of mass of the machine to the centre of the tire contact patch at the front and rear wheels, respectively, $l$ is the total distance between front and rear contact patches, $a$ is the longitudinal acceleration of the vehicle (positive toward the front), $h_{C G}$ is the height of the vehicle centre of mass, $F_{p u s h}$ is the horizontal pushing force of the machine, and $h_{p}$ is the height of that force acting on the machine.

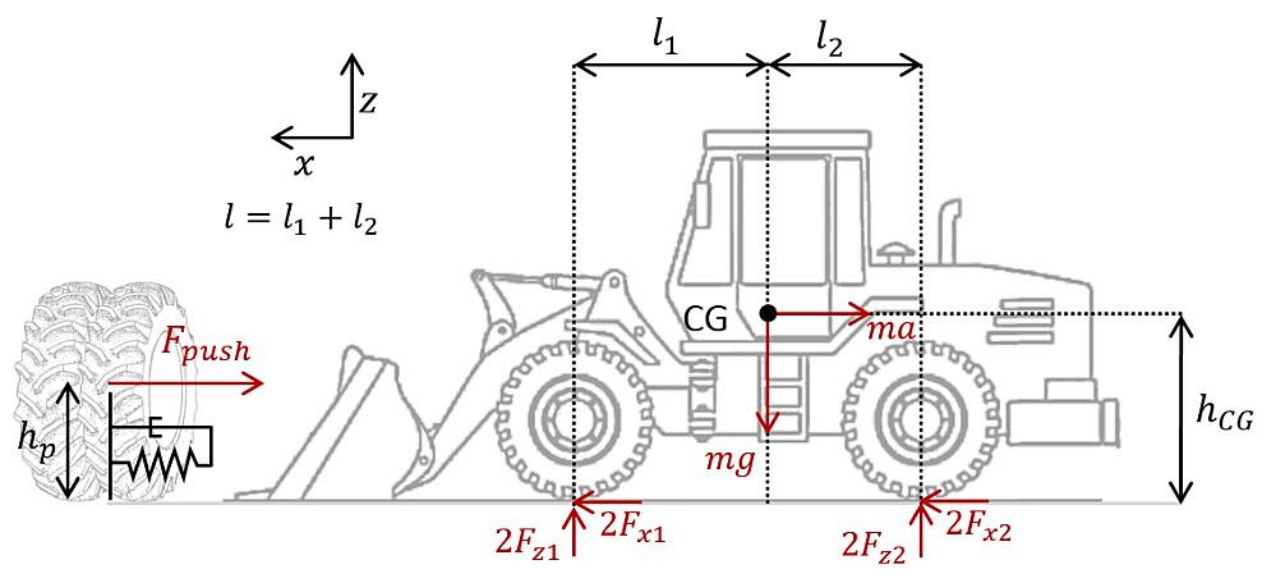

Figure 4. Two-axle vehicle model including vehicle acceleration and pushing action.

As this machine is designed for digging into a work pile of material, it is important that the simulation model be capable of representing a resistive force, as well as the machine reaction to such a force. If the resistive force is caused by a material pile, finding an appropriate model can be relatively complicated, as shown in [25]. For the present work, however, the test condition needed to be more repeatable than digging into a work pile. Therefore, the experimental setup consists of the wheel loader pushing against upright tires, similar to the condition shown in Figure 4. In general, the tires act as a linear spring-damper system in resisting the motion of the wheel loader. For this work, then, the resistive force $F_{\text {push }}$ is calculated as: 


$$
F_{\text {push }}=\left\{\begin{array}{cc}
k_{\text {tires }} x_{\text {tires }}+c_{\text {tires }} \dot{x}_{\text {tires }}, & x_{\text {tires }} \geq 0 \text { and } \dot{x}_{\text {tires }} \geq 0 \\
0, & \text { else }
\end{array},\right.
$$

where $k_{\text {tires }}$ and $c_{\text {tires }}$ are the equivalent spring and damper constant for the upright tires, respectively, and $x_{\text {tires }}$ and $\dot{x}_{\text {tires }}$ are the distance the machine has pushed into the tires and the machine velocity, respectively. The decision structure keeps the tires from exerting a negative resistance force on the vehicle (i.e. pulling it forward). This model should be a very good representation of the resistive force generated in this test setup.

\section{Complete Vehicle Model}

Since the brakes are controlled independently, the braking torque at each wheel is trivial to compute. The engine torque to each wheel, on the other hand, can only be found by examining how the engine torque is distributed to each wheel through the transmission system. For the purpose of this research, it is assumed that the input engine torque into the transfer case of the wheel loader is known. Therefore, other transmission components such as the torque converter are neglected. Furthermore, the wheel loader is a four-wheel drive machine, so all four wheels will see an input torque from the engine via the transmission system. The first component of the transmission system to be modelled is the differential. The present system has two differentials, one at the front and one at the rear. The differentials are responsible for distributing the torque from the driveshaft to the wheels. Through the use of a planetary gear, the torque from the driveshaft is split evenly to each side.

$$
T_{i, L}=T_{i, R}=\frac{1}{2} R_{\text {diff }} T_{D S, i}
$$

where $T_{i, L}$ and $T_{i, R}$ are the input torques at the left and right wheels on axle $i$, respectively, $R_{\text {diff }}$ is the gear ratio from the driveshaft to the differential, and $T_{D S, i}$ is the torque input from the engine at the driveshaft connection to axle $i$.

Along with splitting the torque equally to each wheel, the differential allows the wheels to turn at different speeds. The following is the only relevant equation for relating the wheel speeds at the differential:

$$
\dot{\theta}_{D S, i}=\frac{1}{2 R_{\text {diff }}}\left(\dot{\theta}_{i, L}+\dot{\theta}_{i, R}\right)
$$

In Equation (7), $\theta_{D S, i}$ is the rotational velocity of the driveshaft at axle $i$, and $\theta_{i, L}$ and $\theta_{i, R}$ are the rotational velocities of the left and right wheels on axle $i$. In essence, what the driveshaft sees is the average of the two wheel velocities, scaled by a gear ratio. Equation (6) and (7) are applicable to both the front and rear differentials, which are essentially the same model used for both components.

For the case of the wheel loader, the engine torque is transmitted to the driveshaft via a locked transfer case. This means that the front and rear halves of the driveshaft are actually linked, so that their velocities are equal. The modelling of such a transmission case is more difficult than for an open differential. The process used in this research treats the transfer case, the front and rear differentials, and axles as a rotational dynamic system with the front and rear driveshaft sections acting as very stiff rotational spring/dampers. 
This is consistent with the methodology described in [26]. Through some simplifications of the rotational system, the following relationships are found:

$$
\begin{gathered}
T_{D S, F}=\frac{1}{2} T_{E}-\frac{k_{D S}}{2}\left(\theta_{D S, F}-\theta_{D S, R}\right)-\frac{c_{D S}}{2}\left(\dot{\theta}_{D S, F}-\dot{\theta}_{D S, R}\right) \\
T_{D S, R}=\frac{1}{2} T_{E}+\frac{k_{D S}}{2}\left(\theta_{D S, F}-\theta_{D S, R}\right)+\frac{c_{D S}}{2}\left(\dot{\theta}_{D S, F}-\dot{\theta}_{D S, R}\right),
\end{gathered}
$$

where $T_{E}$ is the torque from the engine into the transfer case, $k_{D S}$ and $c_{D S}$ are the equivalent spring and damper constants, respectively, for the driveshaft sections, $\theta D S, F$ and $\theta D S, R$ are the current angular positions of the front and rear driveshaft sections, respectively, and $\theta_{D S, i}$ and $T_{D S, i}$ are the same as in Equation (6) and (7) above.

If $k_{D S}$ and $c_{D S}$ chosen are sufficiently high, Equation (8) and (9) allow the torque at the front and rear differentials to be significantly different, while maintaining positions and velocities at the front and rear driveshaft sections which are very similar to each other.

\section{Tire Slip Dynamics}

This section describes the motion of the different vehicle components with the proper force and torque inputs. However, the difficulty now becomes related to the tire tractive force to the input torque given to the wheel.

\section{Wheel Slip}

Several different wheel force estimation models exist in the literature but the most widely used is the Magic Formula tire model set forth by [11]. This model relates the longitudinal tractive force of a tire to a parameter known as the slip ratio. Due to tire deformation and irreversible processes such as friction heat loss and material wear, when a torque is being applied to a wheel, these two values do not match. Therefore, an algebraic definition of slip ratio $\kappa$ at wheel $i$ can be defined as in [12]:

$$
\kappa_{i}=\frac{r_{d} \omega_{i}-v_{x}}{v_{x}}
$$

where $r_{d}$ is the dynamic radius of the wheel, $\omega_{i}$ is the rotational velocity of wheel $i$, and $v_{x}$ is the linear velocity of the vehicle. The slip ratio represents the tire deformation mechanism which results in a friction force between the wheel and the road surface.

\section{The Magic Formula Tire Model}

As the slip ratio defines a specific friction condition for a given wheel, in this research, the so-called Magic Formula was used to relate wheel slip to the friction coefficient at that wheel. The longitudinal force was then calculated by multiplying this friction coefficient by the normal force at the tire-road interface.

$$
F_{x, i}=\mu_{x, i}\left(\kappa_{i}\right) F_{N, i},
$$

where $F_{x, i}$ is the longitudinal force at tire $i, \mu_{x, i}$ is the friction coefficient at wheel $i$ (a function of wheel slip $\kappa_{i}$ ), and $F_{N, i}$ is the normal force at wheel $i$. The normal force at each wheel is calculated by using the two-axle model described. 


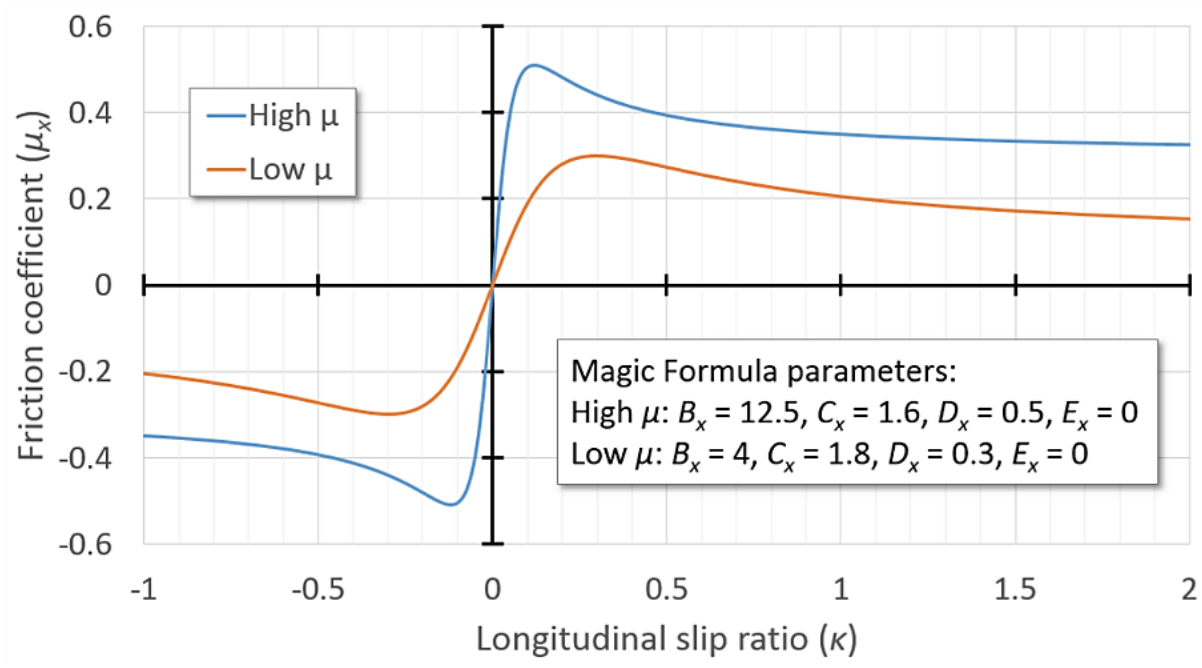

Figure 5. Examples of slip-friction curves defined by the Magic Formula tire model.

The Magic Formula, then, relates to the slip ratio at a given wheel to the friction coefficient at that wheel.

$$
\mu_{x, i}\left(\kappa_{i}\right)=D_{x, i} \sin \left(C_{x, i} \tan ^{-1}\left[B_{x, i} \kappa_{i}-E_{x, i}\left(B_{x, i} \kappa_{i}-\tan ^{-1}\left[B_{x, i} \kappa_{i}\right]\right)\right]\right) .
$$

The terms of this equation ( $B_{x, i}$ through $E_{x, i}$ ) are dimensionless numbers which affect the shape of the resultant Magic Formula curve in various ways. (Of course, $\mu_{x, i}$ and $\kappa_{i}$ are themselves dimensionless terms.) In [27], Pacejka described these terms as follows: $B_{x, i}$ is called the stiffness factor, $C_{x, i}$ is the shape factor, $D_{x, i}$ is the peak factor, and $E_{x, i}$ is referred to as the curvature factor. Two different examples of Magic Formula tire models and their corresponding parameters are shown in Figure 5.

\section{Tire Slip modelling for Low-Velocity System}

The approach described in the previous paragraphs has been widely adopted for describing tire force in-vehicle systems; however, it can easily be seen that it is prone to stability issues in some conditions. The part of this model most susceptible to issues is Equation (10). When the vehicle velocity $v_{x}$ is very low, a small variation in either the vehicle velocity or the wheel velocity will cause a large change in slip ratio. In lowvelocity conditions, the slip calculation becomes too stiff to find an appropriate solution numerically. Furthermore, this model does not allow the vehicle velocity to reach zero, as the slip is undefined in that case. For high-speed automobile simulations, this is not an issue. However, for construction machines such as the wheel loader, much of their operation cycles take place at or around zero velocity. Therefore, a tire slip model needed is stable at very low-velocity. Based on the work done by Bernard [13], the slip ratio definition was changed from the algebraic expression above to a differential equation.

$$
\dot{\kappa}_{i}+\frac{\left|v_{x}\right|}{B} \kappa_{i}=\frac{r_{d} \omega_{i}-v_{x}}{B}
$$


where $B$ is the longitudinal relaxation length of the tire. In general, the relaxation length represents the distance the vehicle must travel before the tire force has risen to a certain percentage of its steady-state value [28].

It can be seen that by including the first-order dynamic with the relaxation length, there is no longer a velocity term in the denominator of the equation. Therefore, the system dynamics with Equation (13) much better behave at low-velocity than its algebraic counterpart.

\section{Resistive Torque and Force Considerations at Low-Velocity}

Many of the difficulties in modelling systems such as the wheel loader considered here arise from the fact that the vehicle works primarily at low-velocities. This is seen quite clearly in sequences which include resistive forces and/or torques which cause the vehicle to reach zero velocity. For the case at hand, these are primarily the resistant forces acting on the linear motion of the vehicle and the braking torque acting on the rotational motion of the wheels as shown in Equation (1) and (2), respectively.

The unique quality which these forces and torques have is that they act opposite the body direction of motion. At low velocities, excessive forces cause an over-correction of the vehicle or wheel velocities, which in turn can create significant oscillations in the modelled system, which are not indicative of what occurs in the real world. They also cause issues when running even simple simulations, and they can impact results negatively. Therefore, a structure which will remove the system's tendency toward these oscillations is needed. Different approaches have been proposed in the past. This includes Bernard [13], who adds a "damping force" to the model at low velocities. Such constructions can yield quite positive results; however, they can add extra complexity to the model and for the purposes of this research, they are unnecessarily complicated.

Instead, the models proposed in this research replace the sgn function in Eqs. (1) and (2) with a saturation block to reduce the force or torque based on the operating condition.

$$
\begin{aligned}
& F_{r, s a t}=F_{r} \operatorname{sat}\left(\frac{v_{x}}{v_{x, s a t}}\right) \\
& T_{B, s a t}=T_{B} \operatorname{sat}\left(\frac{\omega}{\omega_{s a t}}\right)
\end{aligned}
$$

In Eqs. (14) and (15), $F_{r, s a t}$ and $T_{B, s a t}$ represent the resistive force and braking moment, respectively, after they have been adjusted based on the speed of the various system on which they are acting. The terms $v_{x, s a t}$ and $\omega_{\text {sat }}$ represent the saturation limits for the longitudinal linear system velocity and the rotational velocity of a wheel, respectively. The saturation function used in these equations is the standard saturation curve, which limits the output between -1 and 1 . These equations scale down the resistive force or torque when the relevant velocity has an absolute value less than the saturation limit. One important aspect of this structure is that the resistive torque and force are zero when the pertinent velocity is zero. This certainly reflects the actual system performance, as these dissipative processes do not generate loads when there is no motion. The exception to this is the brakes, which have static friction as well as kinetic. The general behaviour of the system by using saturation blocks is shown in Figure 6. Illustrated is a simulation in which a constant braking torque is being applied to the system with some 
initial wheel velocity $\omega>\omega_{\text {sat }}$. As the wheel velocity decreases, it eventually crosses the braking saturation threshold velocity $\omega_{\text {sat }}$. Below this velocity, the braking pressure is scaled down to the wheel velocity. This represents a drastic improvement over the system with the sgn function implemented.

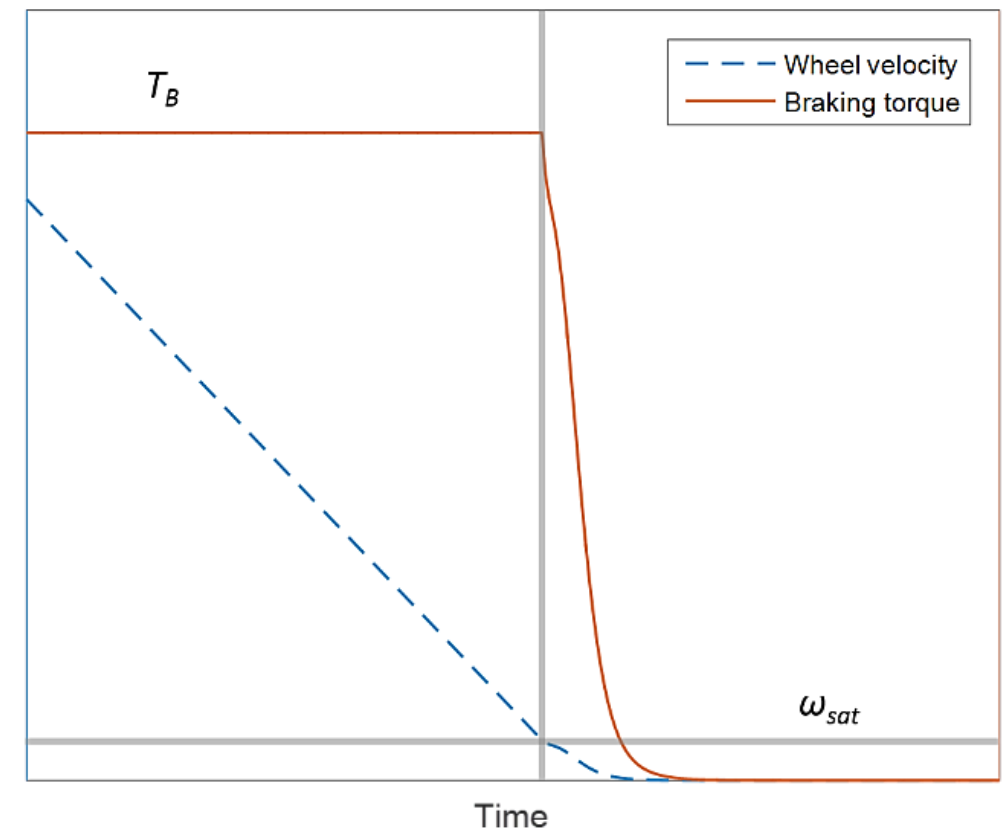

Figure 6. Representative braking manoeuver with a saturation block applied to the braking torque.

\section{RESULTS AND DISCUSSION}

This section outlines the setups for the experimental activities related to this research. These experiments had two main goals. The first is to generate slip-friction data for the construction equipment tires, which have been largely neglected by previous tire modelling investigations. Secondly, these tests provide data with which the simulated system results can be compared. This will serve to help validate the assumptions and simplifications made in constructing the system model.

\section{Slip-Friction Tests}

One of the more important aspects of this work is generating a better estimation of the slip-friction behaviour of the large tires used for the wheel loader. In order to do this, a series of tests was conducted. These tests were based on the work done by Rajamani [14]. By analysing a range of torque commands to the system and their associated responses, the Magic Formula for a given operating condition can be approximated. The tests were also important in that they can be used to validate the system model. The Rajamani method is based on a state estimator which approximates the force at each wheel using measurements of wheel speed, vehicle speed, and vehicle acceleration. The seven different conditions to be tested are shown in Table 1. For this system, there is no direct measurement of the forces at each wheel. Therefore, Rajamani proposed the construction of a state-space system model with a state observer to estimate the force at each wheel. The observer structure is as follows: 


$$
\begin{gathered}
\dot{x}=A x+B u \\
y=C x \\
\dot{\hat{x}}=A \hat{x}+B u+K(y-C \hat{x})
\end{gathered}
$$

Table 1. Test conditions for slip-friction estimate tests.

\begin{tabular}{ccc}
\hline Parameter & Ground Condition & Tire Pressure \\
\hline & Dry concrete & Normal \\
& & Low \\
\cline { 2 - 3 } Values for Test & Light snow on concrete & Normal \\
\cline { 2 - 3 } & & Low \\
\cline { 2 - 3 } & Heavy snow on concrete & Normal \\
\cline { 2 - 3 } & Snow on grass & Normal \\
& & Low \\
\hline
\end{tabular}

with the following state and output vectors were defined:

$$
x=\left[\begin{array}{c}
v_{x} \\
\omega_{F L} \\
\omega_{F R} \\
\omega_{R L} \\
\omega_{R R} \\
F_{x, F L} \\
F_{x, F R} \\
F_{x, R L} \\
F_{x, R R} \\
T_{t o t}
\end{array}\right]=\left[\begin{array}{c}
v_{x} \\
\omega_{i} \\
F_{x, i} \\
T_{t o t}
\end{array}\right] \quad y=\left[\begin{array}{c}
\sum F_{x, i} \\
v_{x} \\
\omega_{F L} \\
\omega_{F R} \\
\omega_{R L} \\
\omega_{R R}
\end{array}\right]=\left[\begin{array}{c}
\sum F_{x, i} \\
v_{x} \\
\omega_{i}
\end{array}\right],
$$

where $x$ is the state vector for the system, $u$ is the input vector, $y$ is the output vector, and $\hat{x}$ is the estimate of the real state-values. $A, B$, and $C$ are the typical state-space system matrices which describe the dynamics of the system, and $K$ is the observer that gains matrix, which controls the convergence of the state estimate to the actual state-values. All terms $v_{x}, \omega_{i}$, and $F_{x, i}$ have the same values as above, and $T_{\text {tot }}$ represents the total torque into the system. It can be seen that the state vector here contains individual values of the force at each wheel $F_{x, i}$ while the output contains only the sum of these four forces (found by using the data acquired by the accelerometer on the system). The other outputs used here are the vehicle velocity and individual wheel velocities (each of which has a dedicated sensor on the machine). Furthermore, the state observer will contain an estimate of the total torque into the system, for which no sensor is installed. The goal is to drive the state estimate $\hat{x}$ to the actual value of the state $x$ by using the available system measurements (or outputs) $y$. 


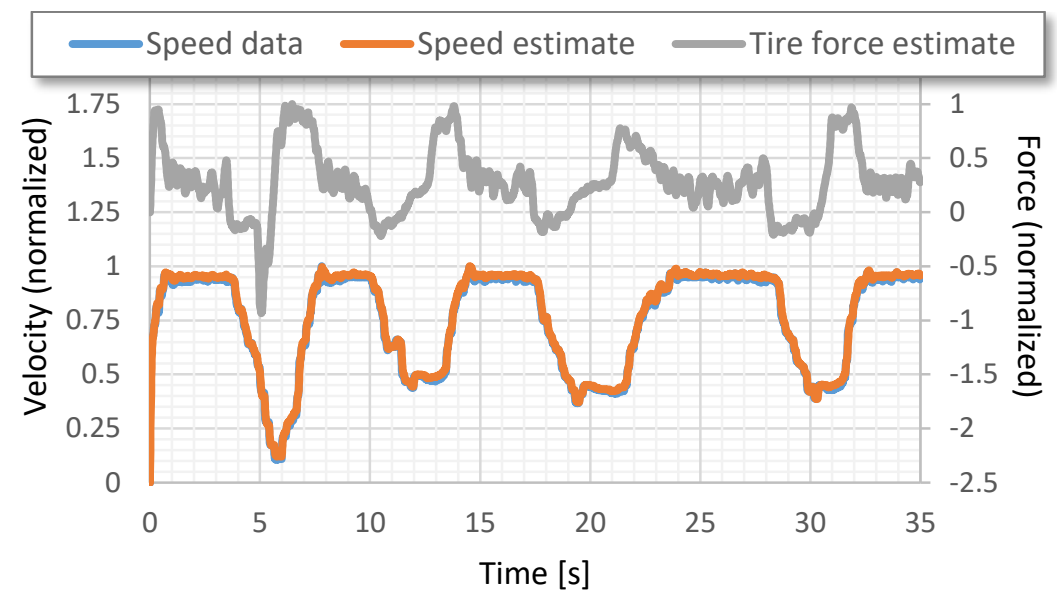

Figure 7. Vehicle velocity data for slip-friction test.

In order to have the best possible representation of a given condition, it is important to test at extreme values of slip. Therefore, the tests were conducted in alternating cycles of rapid acceleration and rapid deceleration as shown in Figure 7. By using the state estimator described above, the force generated at each wheel was estimated and then correlated with the corresponding slip value for that wheel. Figure 7 also demonstrates the reliability of the state estimator used in the tire force estimation. For the vehicle velocity, both the measured data and the estimated values are shown. The estimate matches the data quite well, indicating that the estimator has a good performance for that state. Since all measured states match their estimates very well, it is likely that the unmeasured state estimates are also reliable, assuming a reasonably accurate system model [29].

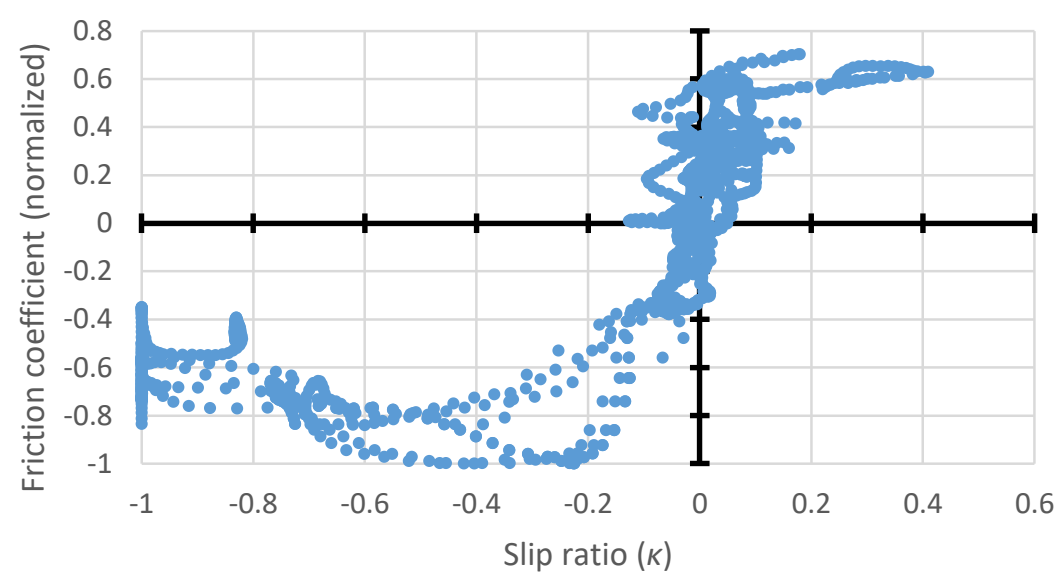

Figure 8. Slip-friction data generated for the wheel loader system (heavy snow on concrete).

\section{Slip-Friction Curve Data}

Figure 8 shows a plot of resulting data for a particular test condition. For the purposes of this research, there is one major assumption which controls the Magic Formula modelling taken from these data points. This is the assumption that the slip-friction relationship has an odd symmetry. This gives two important constraints to the model: first, it will pass through the origin and second, the behaviour with positive slip mirrors that with negative 
slip. With these restrictions, the entire Magic Formula model can be generated from the data shown in Figure 8. In order to properly fit the modelled curve to the data, the Magic Formula parameters $\left(B_{x, i}\right.$ through $\left.E_{x, i}\right)$ were adjusted manually to reduce the mean squared error for the model with respect to the measured data.

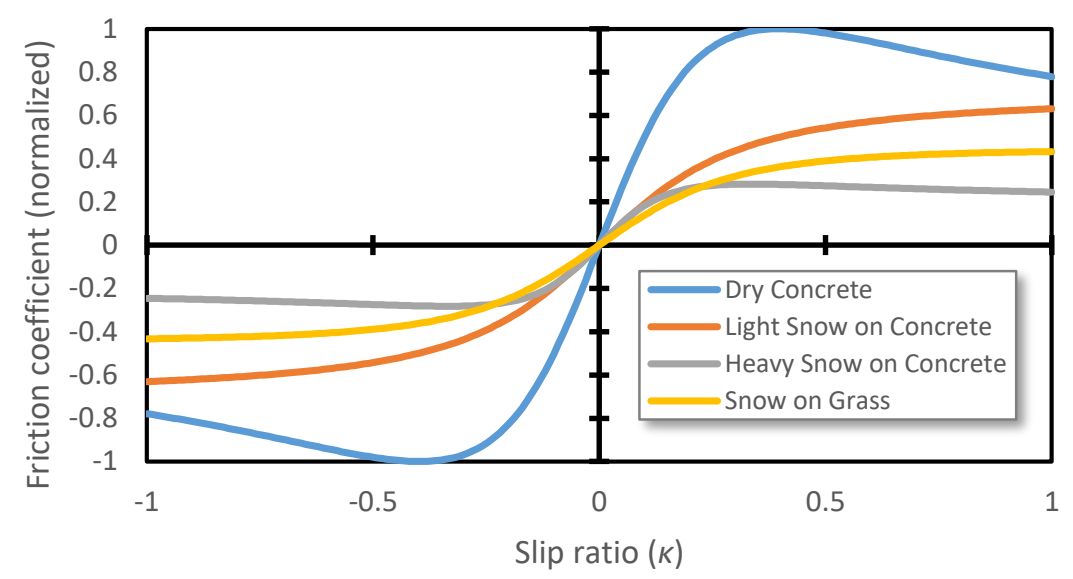

Figure 9. Comparison of slip-friction model road condition.

Data for different road and tire conditions were taken, and curves were fit to each data set. Figure 9 shows a comparison of the resultant curves. This figure has some important results for the current research. First, the trend of road conditions is quite clear and also reasonable to what would be expected for this system. Dry concrete, the best case for a friction force generated between the ground and the wheels, has by far the highest friction coefficient for a given slip ratio. The other cases are relatively similar, with heavy snow having the lowest friction coefficient. Snow on grass was the most variable case but it is more or less comparable to heavy snow on concrete. This data is well in line with what is to be expected from literature, particularly the results listed by Rajamani [14] and [30], where the same behaviour was found for various ground conditions. It should be noted that these slip-friction curves are not intended to match the actual system performance exactly. There are many different potential ground conditions which cannot all be tested in this way, and even within the same condition, there can be a high degree of variability in the resultant friction force. These data were taken primarily to give the investigators a good idea of reasonable values for the friction coefficient of each wheel.

\section{Machine Pushing Tests}

A testing area which contained three large tires on a barrier for the wheel loader to push against (Figure 10a) was constructed. The tires provide a stable and easily-repeatable horizontal force. The wheel loader itself was modified by placing a steel plate across the bucket (Figure 10b), so that the pushing force would be distributed on the tires instead of acting only on the blade and the edge of the bucket. Furthermore, steel plates were placed at the locations where the tires were when conducted the tests. The plates did not provide as much friction with the tires as the concrete, which gave the wheels a better chance of slipping. They also provided a more consistent surface, so that all four wheels were more likely to be in similar ground conditions than they would if they were on the concrete. 


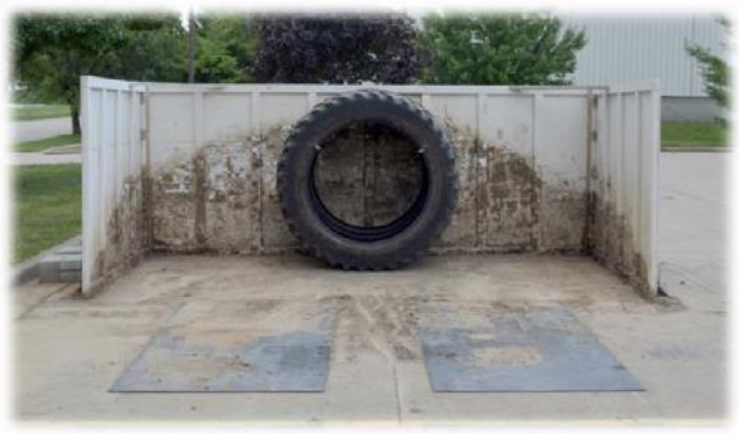

(a)

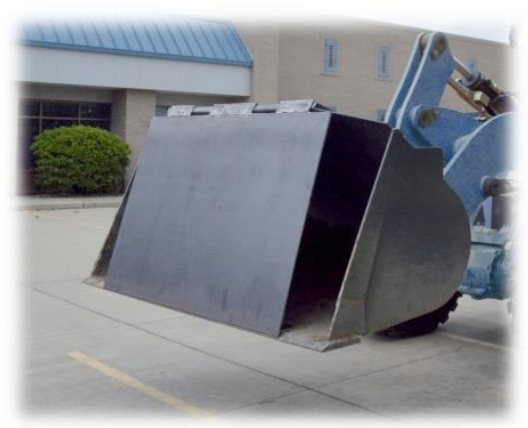

(b)

Figure 10. Experimental setup for resistive force model validation tests, showing (a) the tires for providing resistive force to the wheel loader, and (b) the steel plate installed on the front of the wheel loader bucket for pushing against the tires.

The wheel loader approached the tires at low speed and pushed against them in a low gear while keeping the accelerator at full throttle. This caused the wheels to start slipping. After allowing a few seconds for the system to converge, the accelerator was released and the system ceased pushing against the tires. By analysing the hydraulic pressure in the boom lift cylinder and position of the boom and bucket (which was kept constant for all experiments), it was possible to estimate the pushing force of the machine.

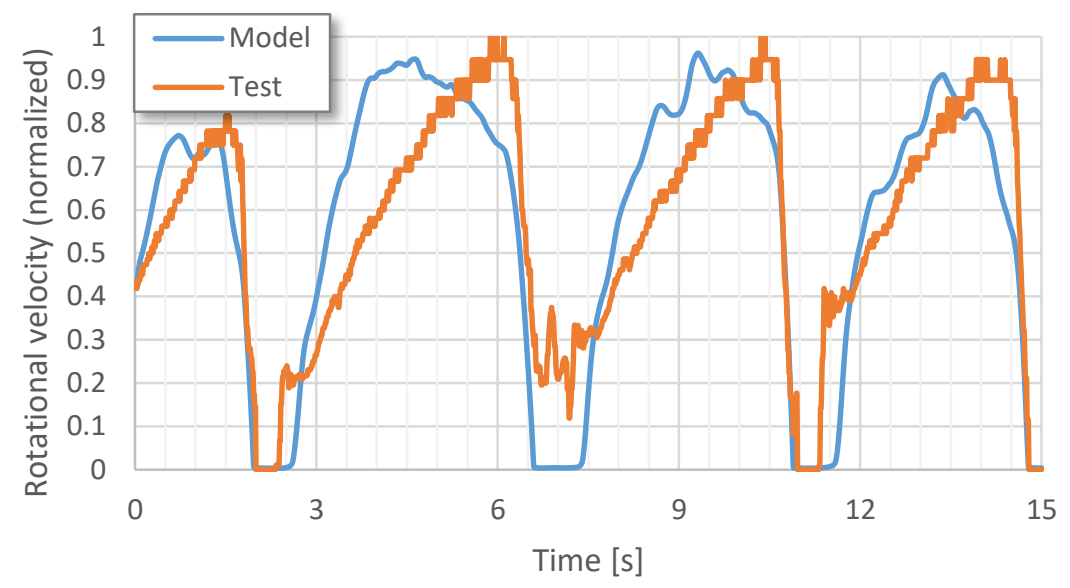

Figure 11. Comparison of simulated and experimental results for wheel velocity.

\section{Tire Model Validation}

With representative slip-friction parameters in the model, it can now be validated by comparing it with the data generated in the experiments. To do this, the inputs to the model (engine torque and braking pressure) must be approximated. Fortunately, the state estimator used to find the force at each wheel also generated an estimate of the input torque to the system. This, along with an estimated braking torque was used as the input to the system model. There were still some differences between the model and the realworld data but this was to be expected. Friction force generation is a stochastic process, where the instantaneous value has a number of different factors, some of which are random in nature. Also, uncertainty on the exact value of the moment of inertia for the wheel rotational dynamics, which included also the driveshaft and axle were present. Therefore, no two cycles will look exactly the same. However, it can be seen from the 
plot in Figure 11 that this simulation model follows the overall trend of the data quite well.

\section{Resistive Force Model Validation}

Another significant aspect of this model is that it is being used for construction equipment which is often used for pushing or digging against a resistive load. Experimental data were generated by pushing against the tires as described. The results of one such test are shown in Figure 12. This figure shows both the estimated pushing force of the reference machine (in grey) and the wheel speeds (in various colours). The force showed what the resistive force model must replicate, while the wheel speeds showed the system model's response to that resistive force. It can be seen that two of the four wheels began slipping and converged to some maximum speed (related to the engine speed and gearing), while the other two wheels dropped down to zero velocity. Simulations incorporating the resistive force model were also conducted. The results of one such simulation are shown in Figure 13. In general, this plot showed that the simulated system had more or less the same behaviour as the real-world system. It should be noted that the wheel forces at each wheel were modified slightly to cause their speeds to diverge as they did in the experimental data.

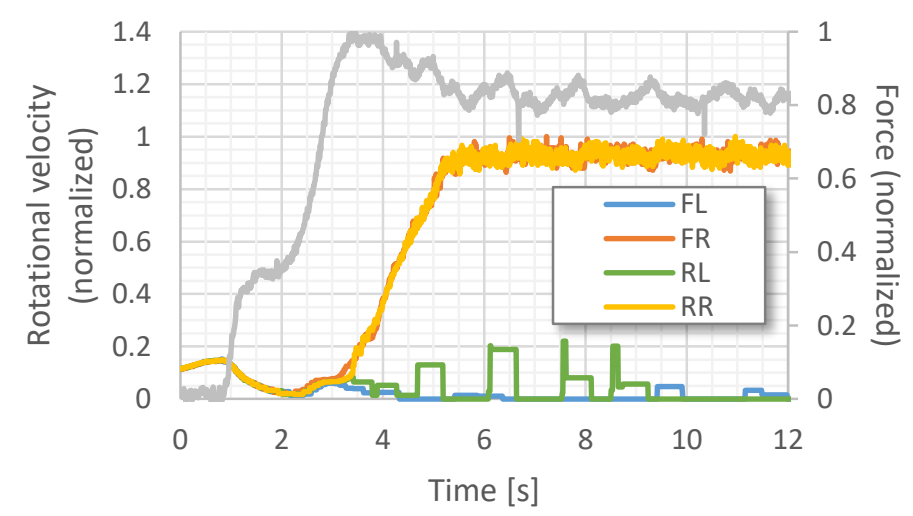

Figure 12. Experimental results for resistive force test.

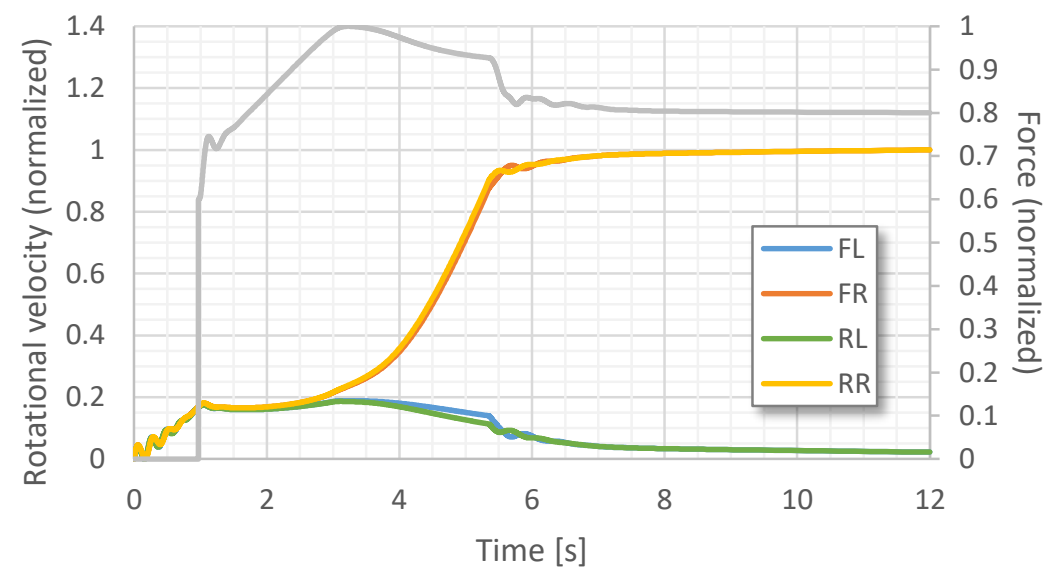

Figure 13. Simulated pushing force using the simplified model.

All in all, the simulation model seemed to replicate, within reason, both the resistive force generated by the tires in experimental tests and the response of the system to such 
a force. In both cases, the wheels initially slowed down but then began slipping up to the maximum value allowed by the transmission speed. And as the wheels begin to slip, the resulting pushing force from the machine was significantly reduced.

\section{CONCLUSIONS}

This paper sets forth the work done in developing and verifying an accurate dynamic model to describe the behaviour of a construction machine in terms of linear velocities of the wheels and the vehicle itself, starting from the given input torque and ground conditions, such as resistance forces (pushing). The dynamic model included considerations for vehicle and wheel dynamics, including weight transfer between axles and the effect of the transmission system. It also included a model for wheel slip behaviour, designed in such a way that the low velocities (typical of construction machines), which can cause serious issues in certain modelling methods, did not have such a negative impact on the simulation. It was further found that it is necessary to model dissipative forces and torques in such a way that the oscillations which are often present when the vehicle reaches zero velocity were eliminated. Experiments were then conducted to determine the slip-friction characteristics of a reference wheel loader. Tests were also conducted in order to examine the validity of the resistive force model included in the system dynamics. All in all, the system model matched the data quite well, and it is being used in further simulations to test various system modifications and operating cycles. By modifying only a few parameters (masses, geometries, etc.), the same analysis can be achieved on a number of different machines without the need for a time-consuming test regimen. For future works, it should be expanded upon easily to include considerations for engine and drivetrain dynamics, braking system dynamics, and more. Future work with this model should include such components, as well as potentially expanding to a system which considers lateral (side to side) motion of the machine, on top of the planar motion already modelled. All of these and more are relatively simple to implement because of the versatile nature of this vehicle model.

\section{ACKNOWLEDGEMENTS}

The authors would like to thank to Purdue University, USA for financial assistance and laboratory facilities.

\section{REFERENCES}

[1] Wong JY. Theory of Ground Vehicles. 4th ed. Hoboken, N.J: Wiley; 2008.

[2] Andreev AF, Kabanau VI, Vantsevich VV. Driveline Systems of Ground Vehicles: Theory and Design Boca Raton, Florida: CRC Press; 2010.

[3] Lichtenheldt R, Barthelmes S, Buse F, Hellerer M. Wheel-Ground Modeling in Planetary Exploration: From Unified Simulation Frameworks Towards Heterogeneous, Multi-tier Wheel Ground Contact Simulation. In: Llagunes J, editor. Multibody Dynamics. Switzerland: Springer; 2016.

[4] Taghavifar H, Mardani A. Off-road Vehicle Dynamics: Analysis, Modelling and Optimization. Switzerland: Springer; 2017.

[5] Nagarkar MP, Vikhe GJ, Borole KR, Nandedkar VM. Active Control of QuarterCar Suspension System Using Linear Quadratic Regulator. International Journal of Automotive and Mechanical Engineering. 2011;3:2180-1606. 
[6] Ramasamy D, Yuan GC, Bakar RA, Zainal ZA. Validation of Road Load Characteristic of a Sub-Compact Vehicle by Engine Operation. International Journal of Automotive and Mechanical Engineering. 2015;9:1820-31.

[7] Clover CL, Bernard JE. Longitudinal Tire Dynamics. Vehicle System Dynamics. 1998;29:231-60.

[8] Gipser M. FTire - the tire simulation model for all applications related to vehicle dynamics. Vehicle System Dynamics. 2007;45:139-51.

[9] Mastinu G, Gaiazzi S, Montanaro F, Pirola D. A Semi-Analytical Tyre Model for Steady- and Transient-State Simulations. Vehicle System Dynamics. 1997;27:221.

[10] Yang S, Chen L, Li S. Dynamics of Vehicle-Road Coupled System Berlin: Springer-Verlag; 2015.

[11] Pacejka HB, Bakker E. The Magic Formula Tyre Model. Vehicle System Dynamics. 1992;21:1-18.

[12] Pacejka HB, Besselink I. Tire and Vehicle Dynamics. 3rd ed. Amsterdam: Elsevier/Butterworth-Heinemann; 2012.

[13] Bernard JE, Clover CL. Tire Modeling for Low-Speed and High-Speed Calculations. 1995 SAE International Congress and Exposition: Society of Automotive Engineers; 1995. p. 85-94.

[14] Rajamani R, Phanomchoeng G, Piyabongkarn D, Lew JY. Algorithms for RealTime Estimation of Individual Wheel Tire-Road Friction Coefficients. IEEE/ASME Transactions on Mechatronics. 2012;17:1183-95.

[15] Bianchi R, Alexander A, Vacca A. Active Vibration Damping for Construction Machines Based on Frequency Identification. SAE Technical Paper. 2016;201601-8121.

[16] Ahmad F, Mazlan SA, Zamzuri H, Jamaluddin H, Hudha K, Short M. Modelling and Validation of the Vehicle Longitudinal Model. International Journal of Automotive and Mechanical Engineering. 2014;10:2042-56.

[17] Jafari M, Mirzaaei M, Mirzaeinejad H. Optimal Nonlinear Control of Vehicle Braking Torques to Generate Practical Stabilizing Yaw Moments. International Journal of Automotive and Mechanical Engineering. 2015;11:2639-53.

[18] Ariff MHM, Zamzuri H, Nordin MAM, Yahya WJ, Mazlan SA, Rahman MAA. Optimal Control Strategy for Low Speed and High Speed Four-Wheel-Active Steering Vehicle. Journal of Mechanical Engineering and Sciences. 2015;8:151628.

[19] Aras MSM, Zambri MKM, Azis FA, Rashid MZA, Kamarudin MN. System identification modelling based on modification of all terrain vehicle (ATV) using wireless control system. Journal of Mechanical Engineering and Sciences. 2015;9:1640-54.

[20] Gillespie TD. Fundamentals of Vehicle Dynamics. Warrendale, PA: Society of Automotive Engineers; 1992.

[21] Rajamani R. Vehicle Dynamics and Control. 2nd ed. New York, NY: Springer; 2012.

[22] Jazar RN. Vehicle Dynamics: Theory and Application. 2nd ed. New York: Springer; 2014.

[23] Schreiber M, Kutzbach HD. Comparison of different zero-slip definitions and a proposal to standardize tire traction performance. Journal of Terramechanics. 2007;44:75-9. 
[24] Schreiber M, Kutzbach HD. Influence of soil and tire parameters on traction. Research in Agricultural Engineering. 2008;54:43-9.

[25] Worley MD, LaSaponara V. Development of a Simplified Load-Cycle Model for Wheel Loader Design. 2006 ASME International Mechanical Engineering Congress and Exposition: ASME; 2006. p. 641-54.

[26] Tinker MM. Wheel Loader Powertrain Modeling for Real-Time Vehicle Dynamic Simulation [Master's thesis]. Iowa City: The University of Iowa; 2006.

[27] Pacejka HB, Besselink IJM. Magic Formula Tyre Model with Transient Properties. Vehicle System Dynamics. 1997;27:234-49.

[28] Cossalter V, Lot R, Massaro M. Motorcycle Dynamics. In: Tanelli M, Corno M, Savaresi SM, editors. Modelling, Simulation and Control of Two-Wheeled Vehicles. Chichester, UK: John Wiley \& Sons, Ltd; 2014. p. 1-42.

[29] The Control Handbook. 2nd ed. ed. Boca Raton, Fla.: CRC Press; 2011.

[30] Ekinci Ş, Çarman K, Kahramanlı H. Investigation and modeling of the tractive performance of radial tires using off-road vehicles. Energy. 2015;93:1953-63. 